\title{
V2G Development on Public Vertical Parking Lot to Support Community Energy Management System
}

\author{
Tinton Dwi Atmaja ${ }^{1, *}$, Vita Susanti ${ }^{1}$, Midriem Mirdanies ${ }^{1}$, and Aam Muharam ${ }^{1,2}$ \\ ${ }^{1}$ Research Centre for Electrical Power and Mechatronics, Indonesian Institute of Sciences, Komp. LIPI Bandung, Jl. \\ Sangkuriang, Gd. 20, Lt. 2, Bandung 40135, Indonesia \\ ${ }^{2}$ Dept. of ASEM, Interdisciplinary Graduate School of Engineering and Sciences, Kyushu University, Global Innovation \\ Center Building, 6 Fl. 6-1, Kasuga-Shi, Fukuoka, 816-8580, Japan
}

\begin{abstract}
Vehicle to grid concept emerged as one solution for harnessing the idle power of parked plug-in hybrid electric vehicle. As the public parking lot had been evolving into the vertical parking lot which has more capacity within the same grounding area, a vehicle to building technology provided more available energy to be shared into the designated area around the parking lot. This paper discussed the development of vehicle to grid into a better concept and architecture by integrating the vertical parking lots one another and also with renewable energy sources (photovoltaic) and sophisticated energy storage system. Several standards were suggested in this paper to ensure a steady performance of vehicle to grid parking. At last, the collaboration scenario was proposed between the parking lot management and plug-in hybrid electric vehicle owner to ensure the technical viability of vehicle to grid implementation for both host and participant. This sharing concept optimized community energy management system as the root segment of the smart city and smart grid delivery system.
\end{abstract}

Keywords: Community energy management system, energy storage system, PHEV, renewable energy, vehicle-to-grid, vertical parking lot.

\section{Introduction}

Climate change and the depletion of fossil fuel lead to emerging research about sustainable energy in the past two decades. Global policies also point to support green technologies including significant portion of attention on green transportation. Therefore, electric vehicles (EV) becomes a high value exploration commodity amongst engineers and researcher. EVs are projected to contribute up to $60 \%$ of total new car sales in the UK by 2030 [1].

Plug-in hybrid electric vehicle (PHEV) in one unique subject for studying EV connection with the power grid as its ability to connect to the grid and conduct vehicle-to-grid (V2G) operation. Equipped with the power cord to connect to the utility grid, PHEV not only capable of charging electricity from the grid but also discharging electricity to the power grid. Upon this capability, V2G can help provide a sufficient energy to powers buildings from their portable battery store, which can be recharged at a different location $[2,3]$.

V2G implementation assists the power grid as backup energy storage when its battery pack is used as demand response and reducing system overload. V2G will also improve power quality, simplifying voltage regulation and reducing power outage, injecting reactive and active power to the grid, and escaping the necessity of building new power plants [4]. Previous research conducted on V2G control such as an autonomous distributed V2G control of gridconnected PHEV and EV by coordinating battery condition, use for vehicle, and power system state [5]. Vehicle to grid concept can be seen in Figure 1 [6]. The PHEV is charging or discharging electricity to the charging infrastructure and later send the power output to the house grid or even to the main utility grid. Renewable energy (RE) sources was also an option to be introduced to the V2G system as RE can increase the stability of the microgrid.

${ }^{*}$ Corresponding author: tinton_dwi@yahoo.com 


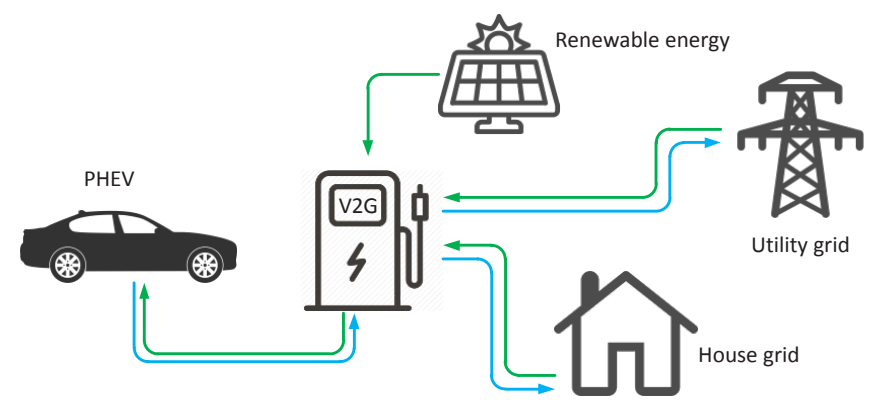

Fig. 1. V2G concept.

Parking garage or parking lot is the most potential facility that can implement V2G concept and deliver the outage power to the nearby building or directly to the utility grid. Evolution of parking lot signs a promising phase to the implementation of V2G. Vertical parking lot have a higher PHEV capacity than on-the-ground parking lot within the same ground area. Higher PHEV capacity means higher electricity capacity to go to the building or to the grid. Figure 2 shows the example of vertical car park [7].

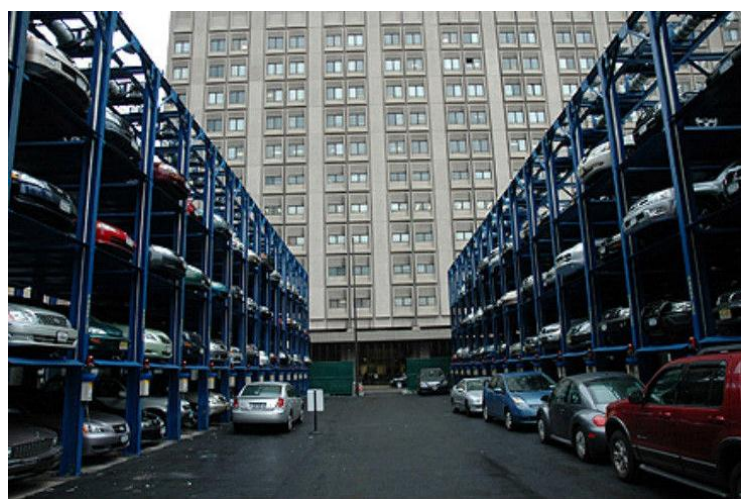

Fig. 2. Vertical parking lot.

Furthermore, V2G parking considerably one of the bases of the emerging concept of community energy management system (CEMS) [8]. Many world class corporation had implemented CEMS to strengthen energy independence and extend the scope of green energy community [9]. Successful CEMS is believed to be one base line which can ensure the steady microgrid among the community which leads to the successfulness of smart grid implementation. The main objective of this work is to study the development of $\mathrm{V} 2 \mathrm{G}$ concept within the sophisticated technology lining up in the worldwide research community. Furthermore, this work should improve the implementation of V2G by adding the key components such as RE integration or energy storage system introduction. The following outcome is that the community can have improvement in their CEMS platform by first improving their parking system.

\section{V2G vertical parking concept}

\subsection{V2G parking concept}

Previous research by Gough et al. [6] develops a data flow and electricity flow in the V2G parking lot. Preceding research [10] also analyze the proper grid structure which can facilitate AC and DC grid for both AC and DC charging mechanism. Figure 3 shows the conceptual model of this work locating the key components, i.e. the location of the parking lot within the building, parked $\mathrm{PHEV}$, charging/discharging infrastructure, building distribution box, utility meter and the utility grid. 


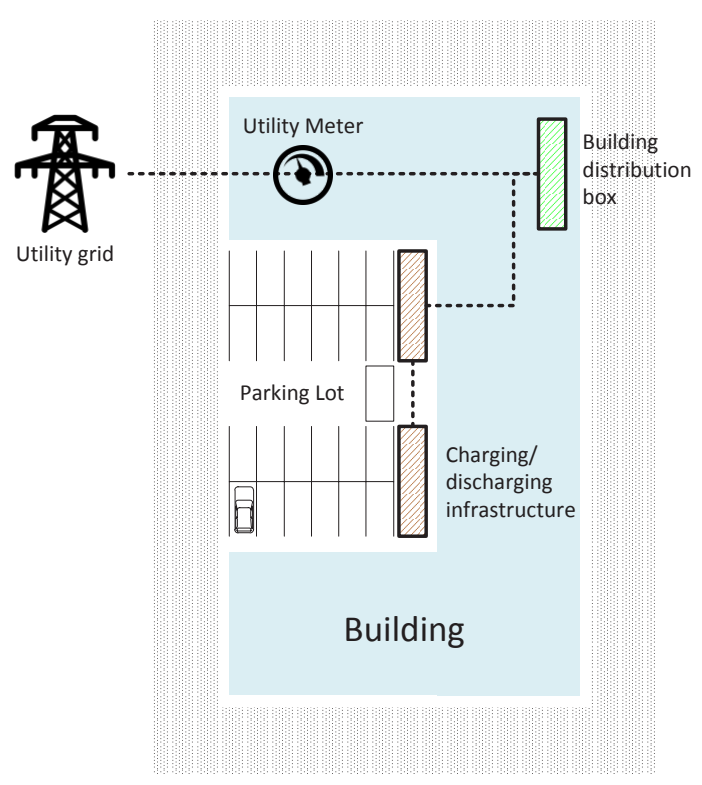

Fig. 3. V2G parking conceptual model illustrating parking location and other key infrastructures.

PHEV in the vertical parking lot connected to the charging infrastructure and further connected into building distribution box. This charging infrastructure is designed to be able to facilitate discharging process from the PHEV into the building/grid. This vehicle-to-building (V2B) process will later be analyzed to bring benefit for both parking lot grid and building grid. The electricity flow also connected into the main utility grid as the main electricity source. The connection is through a bidirectional utility meter to make sure that all incoming and outgoing electricity flow is fairly compensated.

\subsection{Charging infrastructure}

Charging infrastructure specification on this work was based on standard J1772 by the Society of Automotive Engineering (SAE) which classifies AC charging stations into three categories [11]:

i) Level I: charger is on-board and provides $\mathrm{AC}$ voltage $120 \mathrm{~V}$ or $240 \mathrm{~V}$ with a maximum current of $15 \mathrm{~A}$ (standard home outlet), and a maximum power of $3.3 \mathrm{~kW}$.

ii) Level II: charger is on-board and provides $\mathrm{AC}$ voltage $240 \mathrm{~V}$ with a maximum current of $60 \mathrm{~A}$, and a maximum power of $14.4 \mathrm{~kW}$.

iii) Level III: charger is off-board. The charging station provides DC voltage directly to the battery via a DC connector, with a maximum power of $240 \mathrm{~kW}$.

Level III charging station is a fast charger. At the maximum power, level III charging station should be capable of replenishing more than half of the capacity of an EV battery in less than half an hour. Figure 4 shows the schematic of level III charging infrastructure integrated with the building [12]. 


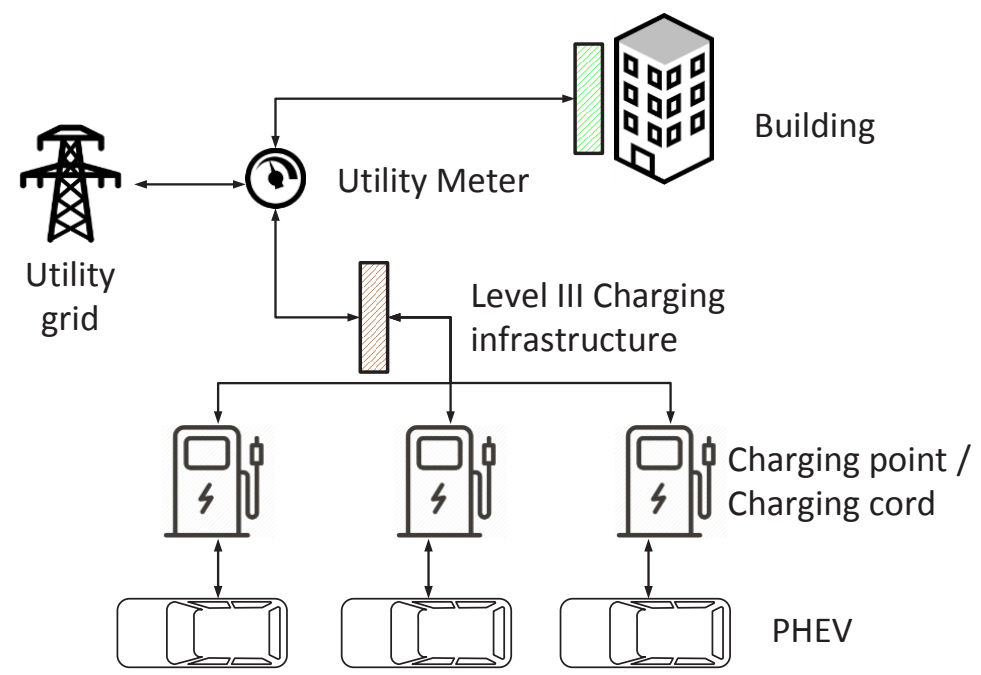

Fig. 4. Schematic of Level III charging infrastructure integrated with a building.

\subsection{Charging scenario}

The Level III charging station operation models can be developed using adopted model from the work of [12, 13] and assimilated with the unique scenarios from [14]. The scenario will be operated at these following points.

i) When the EV come into the parking spot, the EV driver can choose the available charging cord to park their EV. Once the EV plugged, the EV should not move into another charging cord.

ii) If the EV move to another charging cord, and there is a queue in the line, the moved EV will be placed at the end of the queue as if the EV has just arrived.

iii) An EV can only be charged if arriving with at least $80 \%$ battery state of charge but can be immediately charged if there is no queue in the line.

iv) $\mathrm{EV}$ above $80 \%$ can automatically discharge the energy from EV battery into the grid under a specific agreement.

v) Charging will be terminated after the EV battery reach $100 \%$ if there is no queue, and $80 \%$ state of charge if there is a queue

vi) The charging also automatically terminated if the EV disconnected from the charging cord. It is assumed that the driver leaves the parking lot.

\subsection{Related standard}

Standards related to V2G development on public vertical parking can be seen in Table 1 [15]. Those standards can be used as a reference to the specifications, usage, or characteristics of V2G concept, i.e. connectors, socket outlets, safety standards for EV and HEV, etc. For example, the standardization regarding "Connectors, socket outlets, plugs and vehicle inlets" can refer to IEC 621961: 2014, IEC 621962: 2016, IEC 621963: 2014, BS EN 621961: 2014, and JIS D 621963: 2014. The relevant standard about "Road Vehicles Vehicle to Grid Communication" can refer to ISO 151181: 2013, ISO 151182: 2014, and ISO 151183: 2015. This standardization will facilitate companies in developing V2G. Some companies that are doing research related to V2G are Nissan and Mitsubishi, followed by Ford, Tesla, BMW and Honda [16].

Table 1. Various EVs standard related to V2G parking implementation.

\begin{tabular}{ccl}
\hline No & \multicolumn{1}{c}{ Standard } & \multicolumn{1}{c}{ Type and Specification } \\
\hline 1 & IEC 6185123: 2014 & Electric vehicle (EV) conductive charging system Part 23: DC EV charging station \\
2 & IEC 621961: 2014 & $\begin{array}{l}\text { Vehicle connectors, socket outlets, plugs, and vehicle inlets conductive charging of EVs part 1: } \\
\text { general requirements }\end{array}$ \\
3 & IEC 621962: 2016 & $\begin{array}{l}\text { Vehicle connectors, socket outlets, plugs, and vehicle inlets conductive charging of EVs part 2: } \\
\text { contact tube accessories }\end{array}$ \\
4 & IEC 621963: 2014 & $\begin{array}{l}\text { Vehicle connectors, socket outlets, plugs, and vehicle inlets conductive charging of EVs part 3: } \\
\text { contact tube vehicle couplers and dimensional compatibility \& interchangeability requirements for }\end{array}$
\end{tabular}


Table 1. Various EVs standard related to V2G parking implementation (Continued).

\begin{tabular}{|c|c|c|}
\hline No & Standard & Type and Specification \\
\hline & & DC pin \\
\hline 5 & IEC 619801: 2015 & EV WPT systems - part 1: Basic requirements \\
\hline 6 & IEC 6185124: 2014 & $\begin{array}{l}\text { Part } 24 \text { (conductive charging system for EV): To control the DC charging, digitalize } \\
\text { communication between a DC supply based EV charging station and an EV. }\end{array}$ \\
\hline 7 & SAE J 1772: 2016 & Conductive charge coupler for EV and PHEV \\
\hline 8 & SAE J 1773: 2014 & SAE inductively coupled charging for EVs \\
\hline 9 & SAE J J 537: 2016 & Battery storage system \\
\hline 10 & SAE J 1797: 2008 & Packaging of EV battery modules and recommended practice for. \\
\hline 11 & SAE J 1798: 2008 & Performance rating of EV battery modules and recommended practice for \\
\hline 12 & SAE J 2288: 2008 & EV battery modules' life cycle testing \\
\hline 13 & SAE J 2464: 2009 & Safety and abuse testing of EV and HEV rechargeable ESS \\
\hline 14 & SAE J 2758: 2007 & Detail description to determine the maximum available power from a RESS on an HEV \\
\hline 15 & SAE J 2929: 2013 & $\begin{array}{l}\text { Safety standard for EV and HEV propulsion battery systems utilizing lithium based rechargeable } \\
\text { cells }\end{array}$ \\
\hline 16 & SAE J 2936: 2012 & Labelling recommended practice for SAE electrical energy storage device \\
\hline 17 & SAE J 2344: 2010 & EV safety guidelines \\
\hline 18 & SAE J 2894/1: 2011 & Plug-in EV chargers required power quality \\
\hline 19 & SAE J 2894/2: 2015 & Plug-in EV chargers based power quality testing \\
\hline 20 & SAE J 2293/1: 2014 & Functional requirements and system architectures for EVs energy transfer system (part 1) \\
\hline 21 & SAE J 2293/2: 2014 & Communication requirements and network architecture for EVs energy transfer system (part 2) \\
\hline 22 & SAE J 2841: 2010 & Utility factor definitions for PHEV using travel survey data \\
\hline 23 & UL 2202: 2009 & Edition: 2, Detail of EV charging system equipment \\
\hline 24 & UL 22311: 2012 & Edition: 2, general requirement of personnel protection systems for EV supply circuits \\
\hline 25 & UL 22312: 2012 & Edition: 2, particular requirements for protection devices for use in charging systems \\
\hline 26 & UL 2251: 2013 & Edition: 3, couplers, receptacles and plugs for EVs \\
\hline 27 & UL 2734: 2015 & Edition: 3, service plugs and outline for connectors for use with on-board EV charging systems \\
\hline 28 & UL 2871: 2014 & Edition 1: Outline of investigation for and production chargers and EV service \\
\hline 29 & UL 9741: 2014 & Edition 1: Outline of investigation for bidirectional EV charging system equipment \\
\hline 30 & UL 2747: 2012 & Outline of investigation for EV power supplies \\
\hline 31 & BS EN 618511: 2011 & part 1: General requirements for conductive charging system of EVs \\
\hline 32 & BS EN 6185121: 2002 & Requirements of EV to connect an $\mathrm{AC} / \mathrm{DC}$ supply in conducting modes \\
\hline 33 & BS EN 6185122: 2002 & EV charging station based on AC supply \\
\hline 34 & BS EN 621961: 2014 & $\begin{array}{l}\text { General Requirements for vehicle inlets, vehicle connectors, socket outlets, and plugs, for } \\
\text { conductive charging of EVs. }\end{array}$ \\
\hline 35 & $\begin{array}{l}\text { BS DD CLC/TS 504571: } \\
2008\end{array}$ & EVs conductive charging through DC charging station \\
\hline 36 & $\begin{array}{l}\text { BS DD CLC/TS 504572: } \\
2008\end{array}$ & $\begin{array}{l}\text { Detail of communication protocol between EV and off board charger for conductive charging of } \\
\text { EVs. }\end{array}$ \\
\hline 37 & COC GB/T 18487.1: 2015 & Part 1: Details of general requirements for EV conductive charging system \\
\hline 38 & COC GB/T 18487.2: 2001 & EVs requirements for conductive connection to an $\mathrm{AC} / \mathrm{DC}$ supply \\
\hline 39 & COC GB/T 18487.3: 2001 & EV conductive charging system AC/DC EV charging station \\
\hline 40 & $\mathrm{COC} \mathrm{GB/T} \mathrm{20234.1:} 2015$ & Part 1: General requirements for connection set for conductive charging of EVs \\
\hline 41 & $\mathrm{COC} \mathrm{GB/T} \mathrm{20234.2:} 2015$ & Part 2: AC charging coupler for connection set for conductive charging of EVs \\
\hline 42 & COC GB/T 20234.3: 2015 & Part 2: DC charging coupler for connection set for charging conductive charging of EVs \\
\hline 43 & 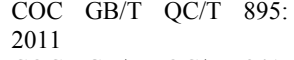 & Detail of EVs on-board conductive charger \\
\hline 44 & $\begin{array}{llll}\mathrm{COC} & \mathrm{GB} / \mathrm{T} & \mathrm{QC} / \mathrm{T} & 841: \\
2010 & \end{array}$ & Conductive charge coupler for EV \\
\hline 45 & JIS D 1304: 2004 & Efficiency test method of an EV charging system \\
\hline 46 & JIS D 6185123: 2014 & Part 23: DC EV charging station based EV conductive charging system \\
\hline 47 & JIS D 6185124: 2014 & $\begin{array}{l}\text { EV conductive charging system part 24: To control the DC charging, digital communication } \\
\text { between EV and a DC EV charging station }\end{array}$ \\
\hline 48 & JIS D 621963: 2014 & $\begin{array}{l}\text { General requirements for vehicle inlets, vehicle connectors, socket outlets, and plugs, for } \\
\text { conductive charging of EVs. }\end{array}$ \\
\hline 49 & ISO 11955: 2008 & ISO/TR 11955 hybrid electric road vehicles guidelines for charge balance measurement \\
\hline 50 & ISO 17409: 2015 & $\begin{array}{l}\text { Electrically propelled road vehicles connection to an external electric power supply safety } \\
\text { requirements }\end{array}$ \\
\hline 51 & ISO 151181: 2013 & $\begin{array}{l}\text { Road vehicles vehicle to grid communication interface part 1: General information and use case } \\
\text { definition }\end{array}$ \\
\hline 52 & ISO 151182: 2014 & $\begin{array}{l}\text { Road vehicles vehicle to grid communication interface part 2: Network and application protocol } \\
\text { requirements }\end{array}$ \\
\hline 53 & ISO 151183: 2015 & $\begin{array}{l}\text { Road vehicles vehicle to grid communication interface part 3: Physical and data link layer } \\
\text { requirements }\end{array}$ \\
\hline
\end{tabular}




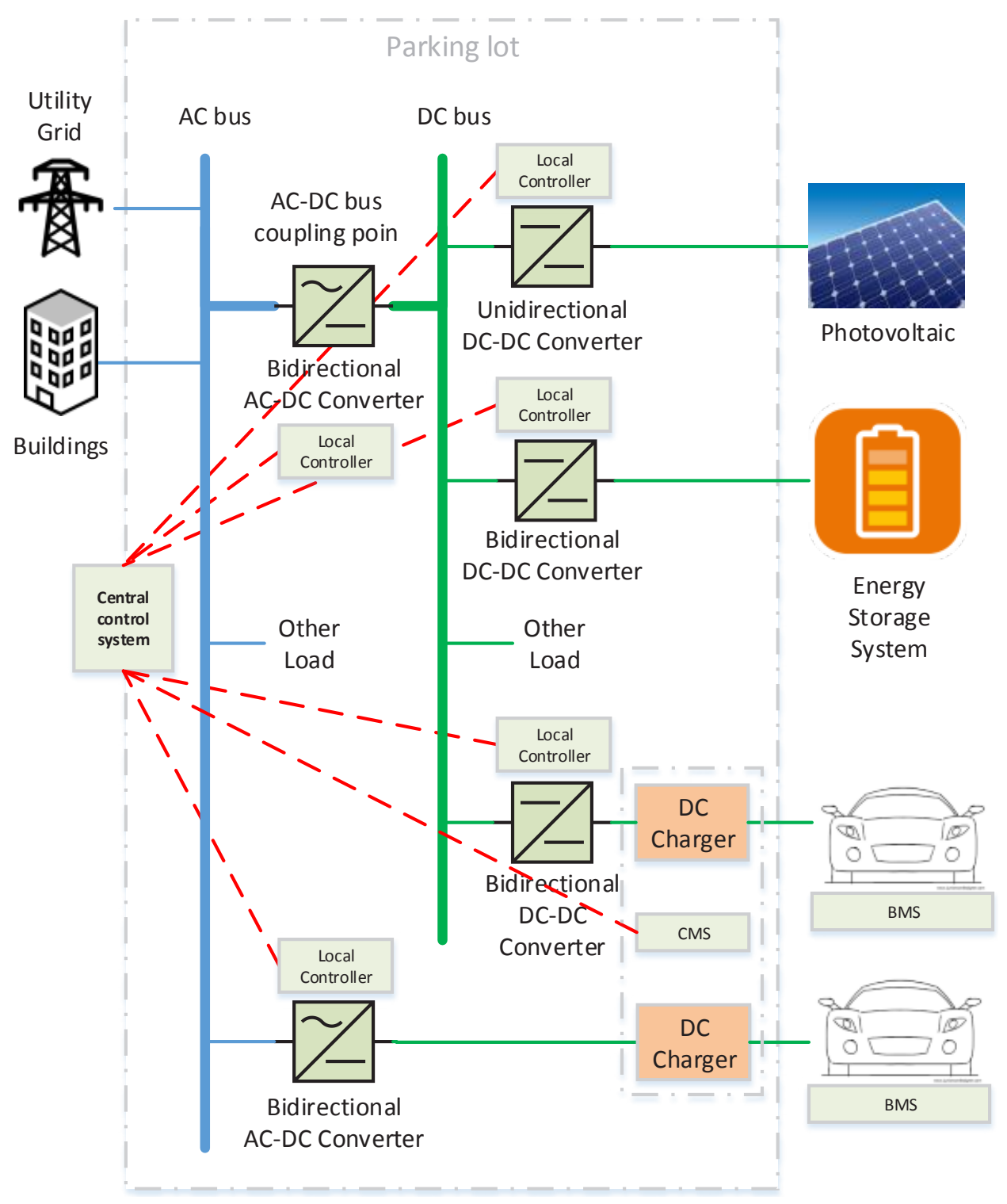

Fig. 5. Control system architecture with PV and ESS for charging mechanism.

\section{V2G parking optimization}

\subsection{Integration with RE and ESS}

The parking lot microgrid could not merely depend on the main utility grid as its primary electricity source. Another backup power source should be installed to reduce electricity cost and diminish the dependency of the utility grid. Solar and wind were the most favorite RE sources that available in the urban area. Solar power could be in the form of concentrated solar power (CSP) or photovoltaic (PV). Previous research [17] was implemented a CSP installation with thermal storage media. However, in several cases, CSP size and efficiency has been overcome by PV panel. 
Figure 5 showed fast charging mechanism with integrated PV and ESS incorporation [14]. There are many energy storage systems (ESS) that could significantly increase the parking lot microgrid stability. Other previous research had also pointed PV as the potential RE integration because of its flexibility such as in vertical façade installment which is suitable for vertical parking lot [18]. Several types of ESSs integration was also discussed in previous research [19].

\subsection{Collaboration possibility}

Collaboration between the owner of the EV and the management of the parking lot was the significant social interaction aspect that would support the success and the continuity of $\mathrm{V} 2 \mathrm{G}$ implementation. Previous research conducted in Université Laval [20] undertake a mutual collaboration between the EV owner and the parking management. The work was highpoints the benefit of vehicle-to-building (V2B) when the EV was connected to the building microgrid via V2G charging infrastructure.

The work investigated a charging scenario that consist of an agreement that allowed the EV to charge for free in exchange for the right of V2G parking management to control the EV energy [20]. The scenario had utilized Battery management system (BMS) onboard of the EV and the charger management system (CMS) inside the V2G parking infrastructure. When the EV connected, the charging infrastructure not only facilitating power exchange but also the data information exchange. The initiation of V2B process would be determined by the CMS unit. CMS other function was including continuous monitoring of BMS data from the connected EV.

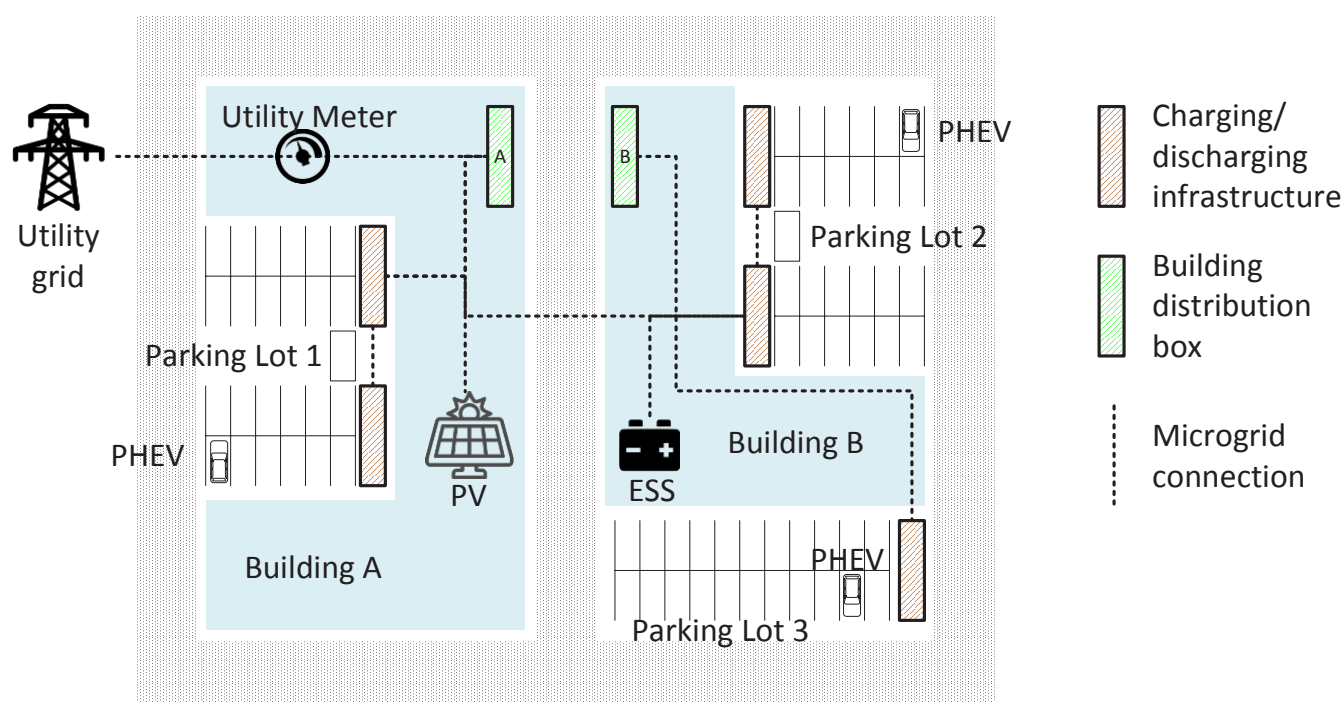

Fig. 6. V2G parking lot integration with buildings, PV, and ESS in detailed location and key infrastructure.

\section{Results and discussion}

\subsection{V2G parking integration with RE and ESS}

The integration of RE sources into the V2G Parking microgrid can be seen in Figure 6. Control configuration of PV to the fast charging system has been detailed in Figure 5. ESS was also introduced to the microgrid with an association of battery management system (BMS) and the charger management system (CMS). In this configuration, the addition of ESS was purposed to assist balancing the microgrid. Bidirectional non-isolated DC-DC converter topologies that can be adopted as the DC chargers were also proposed [14]. Vertical facade installation was considered potential because the vertical parking lot has vertical wall that was rarely used for energy source [10]. Further action would be more focused on developing more efficient scheme to accommodate the integration of the EV into the existing microgrid. The smart energy management should consider numerous proposed sophisticated research like deterministic and fuzzy logic algorithms, genetic and particle swarm optimization algorithms, or online operation algorithm that support the improvement of overall efficiency, control, flexibility and security of the system. 


\subsection{Charging stations integration and building size}

Combining several charging stations and buildings was one of the optimization methods since it could reduce PEV demand charge cost by up to $50 \%$ [12]. Figure 6 contains an illustration where several V2G parking was integrated one another and also linked with several buildings. Using higher power charging station such as $240 \mathrm{~kW}$ charging infrastructure could shrink the refueling time of the EV. However, it is certain that an increase of connected EV to the grid would increase the demand charges of the building.

When the research was focused on building integration, the high demand charges would reduce the sharing demand charges within the building. It could be seen that when the reserved energy was drawn to the high demand charges, the building would receive a minimum energy sharing. However, this integration would be relative to the actual size of the building. If the building was relatively large enough compared to the parking demand charges, then the building would sufficiently share the demand charges. The integration of fast charging infrastructure would also lead to an increase in the switching rates in the larger buildings.

Overall demand charges cost reductions should be affected significantly by the dynamics and size of the building electricity consumption. High load factor buildings had provided little to no cost benefit, and low load factor buildings might provide savings [12]. Integration with larger building could reduce the chance that the EV charged at the time of building reach its maximum demand, but potential saving might shrink as the building size increased. This was, generally, relative to the building utility and operational cost.

\subsection{Collaboration in CEMS}

Collaboration between parking management and EV owner on V2B scenario has been investigated in Université Lavalin [20] to aim the beneficial interaction between both parties. The research was examined the possibility of reducing the electricity bill by comparing the implementation of V2B parking with no-V2B parking scenario. Figure 7 shows that there was energy exchange between the parked EV and the microgrid which was happened in between 400 Prius and 16 MW power grid in Université Lavalin [20]. The energy exchange did not happen constantly every day and was not in the similar amount of energy. Nevertheless, it was concluded that the collaboration was reducing the campus electricity bill by $0.9 \%$ and $1.6 \%$ compared to the no-V2B original scenario [20]. If the collaboration can be implemented in every V2G parking in the community area, it is believed that the electricity bill of the community could be reduced.

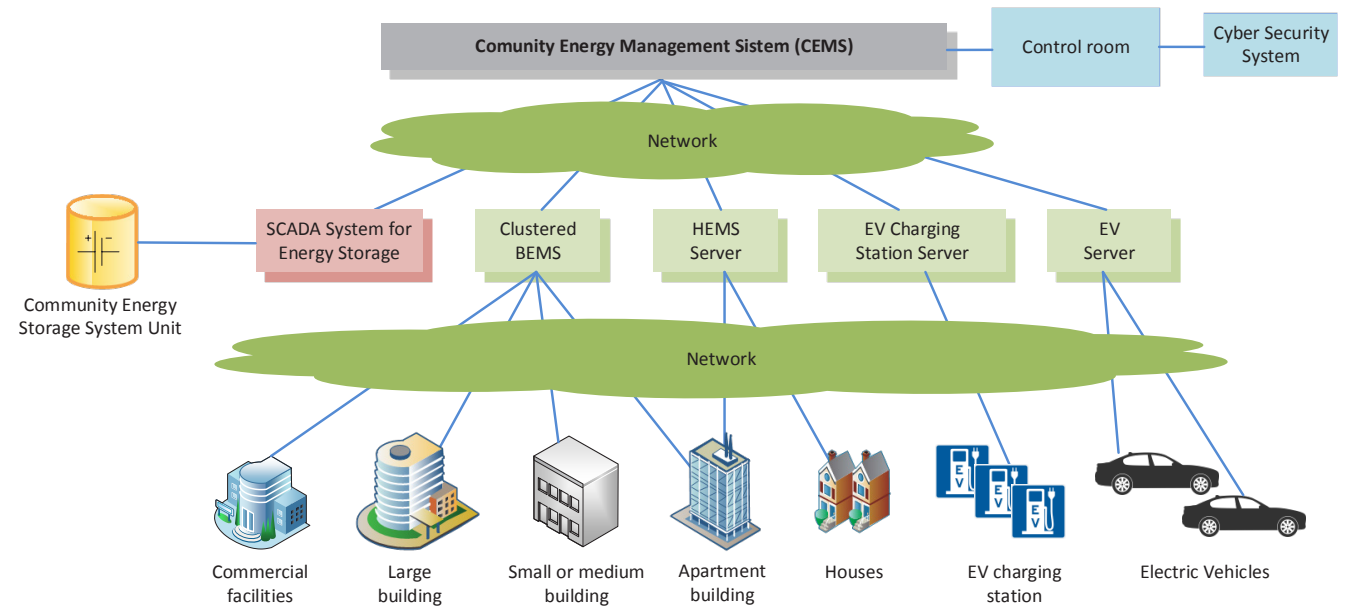

Fig. 8. Brief configuration of CEMS. 


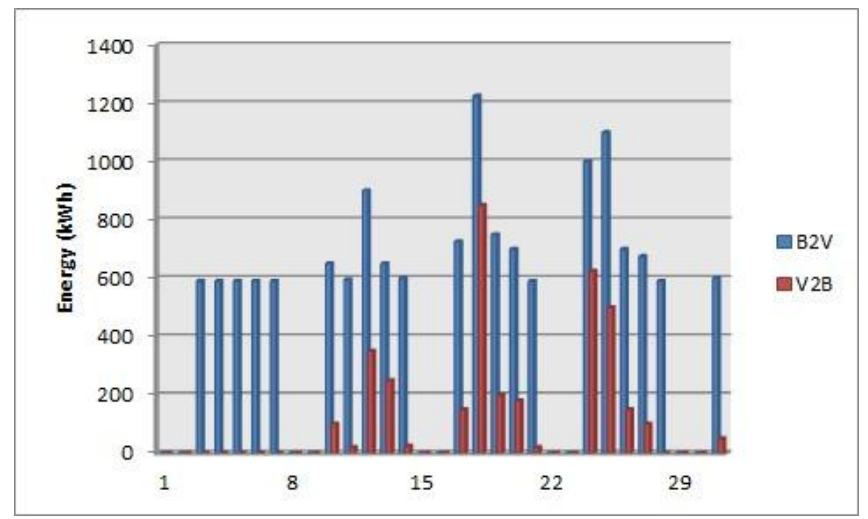

Fig. 7. Energy exchanges between the car and the building for the month of January 2011 at Université Lavalin [20].

Successful collaboration will furthermore support the implementation of CEMS. As mentioned before, there are many corporations that started implementing CEMS in their line of work. Figure 8 is describing the V2G introduction into the CEMS. The concept was adopted from one of CEMS implementation in Yokohama Smart City Project (YSCP) [9]. CEMS project was including the EVs server, EV charging station energy management system, house energy management system (HEMS), building energy management system (BEMS), and energy storage SCADA system.

EV server covered undertakings of dispatched EV within the coverage area of the community or even within the city area. EV server should monitor EVs location, EV power condition, and specific activities such as accidents or depleted EVs. Charging station has its own network and maintained by a server configuring it's charging activity and monitoring the idling potential. Moreover, charging station server should anticipate the possibility of excesses grid demand charges. V2G and V2B implementations were also controlled by this server.

In higher scale, HEMS and BEMS were also integrated to the CEMS server to ensure the grid power demand. These will include small residence, medium house, apartment building, small to medium office, large building, and event commercial facilities within the community. Community energy storage system was installed under specific SCADA system to help the community grid maintain its stability. The whole system was delivered using internet network within the community area. CEMS control room equipped with a designated cyber security system would be a significant addition to the system.

\subsection{Further challenges}

PV integration and ESS introduction have significantly stabilized the microgrid. However, several challenges would emerge as the large deployment of PHEV connected to the power grid. If EV owners were allowed to charge their PHEV whenever they want, power consumption in the parking lot would increase and created a need for higher peak generation capacity [21]. Moreover, increased generation demand due to the high peaks demand would imply to a more expensive system operation cost [22]. As a final consequence, large fleet of PHEV would require a dumb charging approach which require a massive investment in the distribution grid $[23,24]$. Another challenge came from mobility patterns that did not allow EVs to be charged during valley hours. It might increase marginal system cost when intermittent RES generation was low. This would also increase the use of more expensive power generation output and CO2 emissions [25].

\section{CONCLUSION}

This work introduced the implementation of V2G into parking management system. V2G concept facilitated discharging activity from the plugged EV into the building and power grid. This activity would put EV connected to the grid as energy back up which could share its stored energy when the power grid needs it. This sharing activity had ensured the grid stability and reduced charging demand peak. Vertical parking lot provided higher car capacity within same ground area comparing to the conventional on-ground parking lot. This vertical evolution had also provided higher energy back up capacity. Integrating a renewable energy source and introducing dedicated energy storage system had also provided significant peak demand energy absorption. The stable energy capacity from the plugged EV and the integrated RE could ensure a continuous energy share with the building. V2B would emerge a mutual collaboration between EV owner and the building management. Under the specific agreement, the collaboration could reduce electricity cost of the building. The final affection of implementing V2G within the building in the selected area was supporting the success of CEMS which furthermore cement the solid baseline of smart grid implementation. 
The authors would like to express high gratitude to Dian Andriani, Hendri Maja Saputra, and other researchers on Research Centre for Electrical Power and Mechatronics, Indonesian Institute of Sciences, especially in the Industrial Automation Division.

\section{REFERENCES}

1. The Committee on Climate Change. Pathways to high penetration of electric vehicles. [Final Report]. Cambrigde: University of Aberdeen, Ecolane (2013). pp? https://www.theccc.org.uk/wp-content/uploads/2013/12/CCC-EVpathways FINAL-REPORT 17-12-13-Final.pdf

2. C. Guille, G. Gross. Energy Policy, 37(11):4379-4390 (2009). https://www.sciencedirect.com/science/article/pii/S0301421509003978

3. W. Kempton, J. Tomić. J. Power Sources, 144(1):268-279 (2005). https://www.sciencedirect.com/science/article/pii/S0378775305000352

4. F. Khosrojerdi, S. Taheri, H. Taheri, and E. Pouresmaeil. Integration of electric vehicles into a smart power grid: $A$ technical review. Electrical Power and Energy Conference (EPEC) 12-14 October, 2016 (Ottawa, Canada, 2016). IEEE, pp. 1-6 (2016). http://ieeexplore.ieee.org/document/7771784/

5. Y. Ota, H. Taniguchi, T. Nakajima, K. M. Liyanage, A. Yokoyama. An autonomous distributed vehicle-to-grid control of grid-connected electric vehicle. International Conference on Industrial and Information Systems (ICIIS) 28-31 December 2009 (Sri Langka, 2009). IEEE, pp. 414-418 (2010). http://ieeexplore.ieee.org/document/5429826/

6. R. Gough, C. Dickerson, P. Rowley, C. Walsh. Appl. Energy., 192:12-23 (2017). https://www.sciencedirect.com/science/article/pii/S0306261917301149

7. STMY. 3 or 4 level car parking machine manufacturers vertical car storage. [Online] from http://www.myautoparking.com/sale-8227227-3-or-4-level-car-parking-machine-manufacturers-vertical-car-storage.html. [Accessed on 7 July 2017].

8. X. Xu, X. Jin, H. Jia, X. Yu, K. Li. Appl. Energy., 160:231-243 (2015). https://www.sciencedirect.com/science/article/pii/S0306261915010764

9. Toshiba. Development of community energy management system. [Online] from https://www.toshiba.co.jp/rdc/rd/fields/12 e04 e.htm (2010). [Accessed on 31 July 2017].

10. T.D. Atmaja, R. Darussalam, D. Andriani. Vertical facade PV installation to optimize microgrid system on high rise EV parking lot with $A C$ and DC charging station. International Conference on Sustainable Energy Engineering and Application (ICSEEA), 23-24 October 2017 (Jakarta, Indonesia, 2017). IEEE, pp. 164-171 (2018). http://ieeexplore.ieee.org/document/8267703/

11. G. Joos, M. de Freige, M. Dubois. Design and simulation of a fast charging station for PHEV/EV batteries. Electrical Power \& Energy Conference, 25-27 August 2010 (Halifax, Canada, 2010). IEEE, pp. 1-5 (2011). http://ieeexplore.ieee.org/document/5697250/

12. R.J. Flores, B. P. Shaffer, J. Brouwer. Appl. Energy, 191:367-384 (2017). https://www.sciencedirect.com/science/article/pii/S030626191730017X

13. R.J. Flores, B. P. Shaffer, J. Brouwer. Appl. Energy, 169:813-830 (2016). https://www.sciencedirect.com/science/article/pii/S0306261916302082

14. R.H. Ashique, Z. Salam, M.J.B.A. Aziz, A. R. Bhatti, Renew. Sustain. Energy Rev., 69:1243-1257 (2017). https://www.sciencedirect.com/science/article/pii/S136403211631019X

15. F. Ahmad, M.S. Alam, M. Asaad. Sustain. Cities Soc., 35:552-564 (2017) https://www.sciencedirect.com/science/article/pii/S2210670717310557

16. Fleetcarma. The latest in vehicle to grid $(V 2 G)$ charging. [Online] from https://www.fleetcarma.com/latest-vehiclegrid-v2g-charging/ (2017). [Accessed on 07 November 2017].

17. T. D. Atmaja, G. Pikra. Energy Procedia, 32:74-83 (2013). https://www.sciencedirect.com/science/article/pii/S187661021300012X

18. T. D. Atmaja. Energy Procedia, 32:105-114 (2013). https://core.ac.uk/download/pdf/82215540.pdf

19. T. D. Atmaja, Amin. Energy Procedia, 68:429-437 (2015). https://www.sciencedirect.com/science/article/pii/S1876610215005809

20. K. Tanguy, M.R. Dubois, K.L. Lopez, C. Gagné. Sustain. Cities Soc., 26:496-506 (2016). https://www.sciencedirect.com/science/article/pii/S2210670716300439

21. J. Kiviluoma, P. Meibom. Energy, 36(3):1758-1767 (2011). https://www.sciencedirect.com/science/article/pii/S0360544210007590

22. C. Fernandes, P. Frías, L. Olmos, A. Ramos, T. Gómez. Economic impact of plug-in hybrid electric vehicles on power systems operation. The 33rd IAEE International Conference, 6-9 June, 2010 (Rio de Janeiro, Brazil, 2010). https://www.iit.comillas.edu/publicacion/mostrar_publicacion_conferencia.php.en?id=10823

23. J.A.P. Lopes, F.J. Soares, P.M.R. Almeida, Identifying management procedures to deal with connection of Electric Vehicles in the grid. Bucharest PowerTech, 28 June to 2 July, 2009 (Bucharest, Romania, 2009). IEEE, pp. 1-8 (2009). http://ieeexplore.iee.org/document/5282155/ 
24. L.P.Fernandez, T.G.S. Roman, R. Cossent, C. Mateo Domingo, P. Frias. Assessment of the Impact of Plug-in Electric Vehicles on Distribution Networks. Trans. Power Syst., 26(1):206-213 (2011).

http://ieeexplore.ieee.org/document/5471115/

25. C. Fernandes, P. Frías, J. M. Latorre. Appl. Energy, 96:194-202 (2012). https://www.sciencedirect.com/science/article/pii/S0306261911007641 